\title{
Pulmonary hypertension in a patient with Abt-Letterer- Siwe syndrome and episodes of HAPE
}

\section{To the Editors:}

Abt-Letterer-Siwe syndrome, which is a disseminated form of Langerhans' cell histiocytosis ( $\mathrm{LCH})$, was diagnosed in the patient at the age of 6 months, affecting the inguinal lymph nodes and skin, and recurred at the age of 21 yrs, affecting lungs and arcus pubis [1]. After two chemotherapy sessions, including vinblastine, etoposide and corticosteroids, complete remission was reached. At the age of 25 yrs the first highaltitude oedema occurred during a ski vacation at 2,400 m followed by the second episode 3 yrs later at an altitude of $2,800 \mathrm{~m}$, which was confirmed by chest radiograph. After the second episode of high-altitude pulmonary oedema (HAPE) the patient was referred to the Division of Sports Medicine at the University Hospital of Heidelberg, Heidelberg, Germany, where he was assessed as described before [2]. Estimated systolic pulmonary artery pressure $(P \mathrm{pa})$ was normal at rest (25 mm Hg), increased to $50 \mathrm{mmHg}$ after $30 \mathrm{~min}$ (oxygen saturation $74 \%$ ) and to $60 \mathrm{mmHg}$ (saturation 65\%) after $90 \mathrm{~min}$ of exposure to normobaric hypoxia (inspiratory oxygen fraction $\left(\mathrm{FI}, \mathrm{O}_{2}\right)$ of $\left.12 \%\right)$. Stress Doppler echocadiography revealed systolic $P$ pa values up to $95 \mathrm{mmHg}$ during normoxic exercise of $125 \mathrm{~W}$. The patient had a normal systemic blood pressure at rest and during exercise and no signs of left ventricular dysfunction. Maximum exercise capacity was $1.95 \mathrm{~W} \cdot \mathrm{kg}^{-1}$ (70\% of normal), maximum oxygen consumption $\left(V^{\prime} \mathrm{O}_{2}\right)$ was $2.6 \mathrm{~L} \cdot \mathrm{min}^{-1}$ (80\% of normal), body plethysmography showed patterns of obstructive and restrictive lung disease (airway resistance $158 \%$ of normal, forced expiratory volume in $1 \mathrm{~s} /$ forced vital capacity (FEV1/FVC) ratio was $60 \%$ (70\% predicted), vital capacity was $70 \%$, residual volume $166 \%$, total lung capacity $95 \%$ ) and a reduced diffusion capacity for carbon monoxide ( $48 \%$ pred). Despite shortness of breath, the patient was able to jog twice a week over 5$10 \mathrm{~km}$. We suggested that besides his changes in lung function parameters, early pulmonary vascular changes may have led to the patient's elevated Ppa during hypoxia and exercise and may have contributed to his impaired exercise capacity. Recommendations for right heart catheterisation and regular control visits were not followed by the patient.

At the age of $35 \mathrm{yrs}$, exercise performance had further decreased (workload at maximum exercise was 51\% pred and peak $V^{\prime} \mathrm{O}_{2} 61 \%$ pred) while lung function and gas exchange were largely unchanged compared with 7 yrs previously. The patient had an airway obstruction grade II with FEV1 values of $50 \%$ pred, which remained stable during further follow-up. FEV1/FVC ratio was $51 \%$ and airway resistance was $158 \%$ of normal. The total lung capacity was slightly reduced $(6 \mathrm{~L}$, $90 \%$ ), the diffusion capacity of the lung for carbon monoxide was considerably reduced (55\% of normal; transfer coefficient of the lung for carbon monoxide measured 59\%) and raw values were $158 \%$ of normal and remained unchanged during follow-up.
Computed tomography (CT) scan of the lung showed typical residual findings of histiocytosis with irregular cysts in the upper and middle zones of the lung. Thoracoscopic biopsy of the lung revealed no signs of a relapse of $\mathrm{LCH}$, but residual multifocal scars of the lung tissue and diffuse histological changes of the pulmonary arteries with medial and subintimal wall thickening obstructing the vessels. The pathohistological findings were performed in Heidelberg and were reviewed in the University of Bochum (Bochum, Germany). Dilatation of the right ventricle with moderately reduced contractility was documented by echocardiography. Right heart catheterisation revealed an early stage of pulmonary hypertension (table 1) and medical therapy with sildenafil $20 \mathrm{mg}$ three times per day was initiated. This treatment was tolerated well and improved exercise capacity, right ventricular pump function and $P$ pa within a follow-up time of 18 months.

This is the first report of recurrent episodes of HAPE at moderate altitude in a patient with Abt-Letterer-Siwe syndrome who developed a symptomatic chronic pulmonary hypertension a few years later. The lung histology of the patient revealed diffuse pulmonary vasculopathy, including intimal hypertrophy and obstruction of pulmonary arteries as previously described in patients with $\mathrm{LCH}$ [3]. We cannot exclude that the coexistence of recurrent HAPE and the development of pulmonary hypertension in our case was coincidental. However, the histological and clinical findings suggest that both the HAPE episodes and the progression to a manifest chronic pulmonary hypertension are due to the pulmonary vasculopathy, which is common in LCH.

The clinical findings indicate that the pulmonary hypertension in this patient is related to LCH (Dana Point Category V) rather than to group III. The patient had histologically proven $\mathrm{LCH}$ with involvement of the lung at childhood. Although there was no histological evidence of an active relapse of $\mathrm{LCH}$ in the

TABLE 1 Right heart catheterisation

\begin{tabular}{|c|c|c|c|c|c|}
\hline & \multicolumn{3}{|c|}{$P$ pa } & \multirow[t]{2}{*}{ Ppcw } & \multirow[t]{2}{*}{ PVR } \\
\hline & Systolic & Diastolic & Mean & & \\
\hline At rest & 42 & 20 & 27 & 12 & 462 \\
\hline $2 \mathrm{~L} \mathrm{O}_{2}$ & 41 & 21 & 28 & 12 & 492 \\
\hline Supine & 48 & 23 & 31 & 15 & 492 \\
\hline $25 \mathrm{~W}$ & 58 & 25 & 36 & 16 & 391 \\
\hline $50 \mathrm{~W}$ & 60 & 25 & 37 & 13 & 270 \\
\hline Iloprost & 32 & 12 & 19 & 9 & 222 \\
\hline
\end{tabular}

Ppa: pulmonary arterial pressure; $P$ pcw: pulmonary capillary wedge pressure; PVR: pulmonary vascular resistance. 
biopsy during follow-up, the CT scan of the lung showed typical residual findings of histiocytosis with irregular cysts in the upper and middle zones of the lung. Histopathological observations have suggested that pulmonary vascular involvement is a central process in advanced pulmonary histiocytosis [4] and is assumed in all patients with LCH [5]. In addition to the characteristic bronchiolocentric inflammation with aggregates of Langerhans' cells and other inflammatory cells, and fibrosis [4], widespread vascular abnormalities are found in the majority of cases [3]. Patients with pulmonary histiocytosis have revealed proliferative vasculopathy of muscular arteries and veins, with prominent venular involvement [3]. Partial occlusion of vessels can be caused by Langerhans' cell granuloma, which infiltrates the walls of small and mediumsized pulmonary arteries, whereas medial and subintimal wall thickening may occur in areas uninvolved with pulmonary histiocytosis nodules [4]. In our patients, mainly the pulmonary arterioles were involved.

In contrast to patients with chronic obstructive pulmonary disease (COPD) or interstitial pulmonary fibrosis, pulmonary haemodynamics were unrelated to lung function variables in $\mathrm{LCH}$ [3]. Furthermore, to the best of our knowledge, to date no study has previously described an association of HAPE episodes with COPD or a restrictive pulmonary disease. Therefore, we conclude that the central process was the vascular process and the pulmonary hypertension in our case belonged to group $\mathrm{V}$ of the Dana Point classification.

It is well known that an obstruction of the pulmonary vascular bed can cause HAPE at relatively low altitudes. Several cases with unilateral absent pulmonary artery and development of HAPE at altitudes between 2,000 and 2,800 $\mathrm{m}$ have been reported [6]. Atypical cases of HAPE caused by pulmonary embolism have also been observed (unpublished data; P. Bärtsch, personal communication).

The pathogenesis of HAPE is still debated. The prevailing concept suggests that HAPE results from intense and uneven hypoxic vasoconstriction, leading to excessive pressure and oedema formation in the capillaries of overperfused lung regions $[7,8]$. The pulmonary vascular changes as documented by biopsy in our patient may predispose to HAPE by the following mechanism. The reduction of cross-sectional area and a random distribution of occluded or narrowed arterioles enhance uneven perfusion, while occlusion of venules, which was less prominent in our patient, raises capillary pressure by increasing post-capillary resistance.

At the time when HAPE occurred in our patient, systolic Ppa was normal at rest and only abnormally elevated during prolonged hypoxia; normoxic exercise has also been documented in other HAPE-susceptible individuals [2]. The deterioration of our patient's performance over the last 5 yrs is most likely attributed to the progression of pulmonary hypertension to a manifest disease. The pulmonary vasculopathy progressed, although the histiocytosis appeared to be inactive in specimens obtained by lung biopsy, and pulmonary function was almost unchanged. As far as we know there is only one further case report of a patient who had episodes of HAPE and was diagnosed with mild pulmonary arterial hypertension (PAH) 3 yrs later [9]. In this otherwise healthy
43 yr-old-female, PAH was diagnosed by elevated mean $P$ pa during exercise up to $34 \mathrm{mmHg}$ with normal pressures at rest. Referring to the new diagnostic criteria, the patient would no longer be classified as manifest PAH [10]. In our case, $P$ pa and pulmonary vascular resistance have been elevated at rest.

The therapy with sildenafil was well tolerated in our patients. This is in some contrast to a previous observation. In some patients with $\mathrm{LCH}$ and prominent venous involvement, pathohistological changes are similar to veno-occlusive disease. It has been reported that an acute pulmonary oedema occurred in two patients with pulmonary histiocytosis treated with pulmonary hypertension-specific medication. The authors suggested that in these patients histiocytosis caused pulmonary venous obstruction [4]. However, the episodes of HAPE in our patient were not related to medical therapy. Furthermore, it is also unlikely that the vascular alterations seen in our patients were related to the treatment with vinblastine or etoposide.

In conclusion, this is the first case report of pulmonary vascular manifestations of LCH leading to recurrent HAPE episodes at an early stage before developing into manifest pulmonary hypertension. The case suggests that HAPE may be an early clinical manifestation of a chronic progressive pulmonary vasculopathy that can be successfully treated by phosphodiesterase inhibitors.

\section{Corvinus*, P. Bärtsch ${ }^{\#}$, C. Dehnert ${ }^{\#}$, F.J. Herth* and E. Grünig*}

*Dept of Pneumology, Thoraxclinic, and "Dept of Sports Medicine, University of Heidelberg, Heidelberg, Germany.

Correspondence: E. Grünig, Center of Pulmonary Hypertension at the Thoraxclinic, University Hospital Heidelberg, Amalienstrasse 5, 69126 Heidelberg, Germany. E-mail: ekkehard.gruenig@thoraxklinik-heidelberg.de

Statement of Interest: None declared.

Acknowledgements: The authors would like to thank the pathologists Prof. Dr P. Schnabel (University of Heidelberg, Germany) and Prof. Dr K-M. Müller (University of Bochum, Germany) for their excellent pathohistological workup and analysis.

\section{REFERENCES}

1 Salotti A, Nanduri V, Pearce MS, et al. Incidence and clinical features of Langerhans cell histiocytosis in the UK and Ireland. Arch Dis Child 2009; 94: 376-380.

2 Grünig E, Mereles D, Hildebrandt W, et al. Stress doppler echocardiography for identification of susceptibility to high altitude pulmonary edema. J Am Coll Cardiol 2000; 35: 980-987.

3 Fartoukh M, Humbert M, Capron F, et al. Severe pulmonary hypertension in histiocytosis X. Am J Respir Crit Care Med 2000; 161: 216-223.

4 Travis WD, Borok Z, Roum JH, et al. Pulmonary Langerhans cell granulomatosis (histiocytos is $\mathrm{X}$ ): a clinicopathologic study of 48 cases. Am J Surg Pathol 1993; 17: 971-986.

5 Ryu JH, Krowka MJ, Pellikka PA, et al. Pulmonary hypertension in patients with interstitial lung diseases. Mayo Clin Proc 2007; 82: 342-350.

6 Hackett $\mathrm{PH}, \mathrm{Creagh} \mathrm{CE}$, Grover RF, et al. High altitude pulmonary edema in persons without the right pulmonary artery. $N$ Engl $J$ Med 1980; 302: 1070-1073. 
7 Bärtsch $\mathrm{P}$, Mairbäurl $\mathrm{H}$, Maggiorini $\mathrm{M}$, et al. Physiological aspects of high-altitude pulmonary edema. J Appl Physiol 2005; 98: 1101-1110.

8 Dehnert C, Risse F, Ley S, et al. Magnetic resonance imaging of uneven pulmonary perfusion in hypoxia in humans. Am J Respir Crit Care Med 2006; 174: 1132-1138.

9 Naeije R, De Backer D, Vachiéry JL, et al. High-altitude pulmonary edema with primary pulmonary hypertension. Chest 1996; 110: 286-289.
10 Galié N, Hoeper MM, Humbert M, et al. Guidelines for the diagnosis and treatment of pulmonary hypertension. The Task Force for the Diagnosis and Treatment of Pulmonary Hypertension of the European Society of Cardiology (ESC) and the European Respiratory Society (ERS), endorsed by the International Society of Heart and Lung Transplantation (ISHLT). Eur Respir J 2009; 34: 1219-1263.

DOI: $10.1183 / 09031936.00200409$

\section{Pulmonary alveolar proteinosis associated with Epstein-Barr virus infection}

To the Editors:

We report the case of a 3-yr-old male who became unwell over a period of months. He was found to have pulmonary alveolar proteinosis (PAP), confirmed by computed tomography (CT) scan, bronchoalveolar lavage fluid and lung biopsy. Histology from the lung biopsy also revealed a high load of Epstein-Barr virus (EBV). EBV DNA was also found in the blood samples by PCR. We believe this is the first case of PAP caused by EBV reported in the literature.

A 3-yr-old male, born at 34 weeks gestation, had transient infantile hypothyroidism. He had been discharged from an endocrinology clinic at 2 yrs of age, but re-presented with failure to thrive. He was the second twin of consanguineous Asian parents, who had lived in the UK for 15 yrs. They had three healthy older daughters. On examination, he was tachypnoeic and tachycardic with signs of respiratory distress. He had persistent hypoxaemia despite high-flow oxygen and was admitted to the Paediatric Intensive Care Unit (Sheffield Children's Hospital, Sheffield, UK). Chest radiography showed bilateral air-space opacification, and he was administered i.v. cefuroxime. Within $12 \mathrm{~h}$, his condition deteriorated and endotracheal mechanical ventilation was commenced. An initial diagnosis of pneumonia, possibly due to an atypical organism or tuberculosis, was made and the antibiotic spectrum was widened to include clarithromycin, cotrimoxazole, clindamycin, rifampicin, isoniazid, pyridoxine, pyrazonamide and fluconazole.

A blind tracheobronchial lavage was performed by the intensive-care staff. The aspirated fluid was reported to be clear and a silver stain was negative for Pneumocystis jiroveci, but rhinovirus was detected by PCR; there was no significant bacterial growth. The lavage was negative for influenza A and $B$, parainfluenza 1, 2, and 3, respiratory syncytial virus, and adenovirus. A respiratory review 1 week after admission raised the question of interstitial lung disease, and a high-resolution CT scan of the chest and a lung biopsy were performed.

The CT scan (fig. 1) showed severe, bilateral, widespread interstitial opacities with generalised, interlobular septal thickening and ground-glass opacities, with bilateral dependent atelectasis. The changes were felt to be nonspecific, although suggestive of PAP.
PCR blood tests for EBV were positive, with "raised" EBV immunoglobulin (Ig)M and IgG (levels not reported). The histology of the lung biopsy (fig. 2) showed alveolar spaces containing Periodic acid-Schiff-positive eosinophilic proteinaceous granular material, foamy macrophages and cholesterol clefts. In the absence of inflammation and/or interstitial fibrosis, these features were consistent with PAP. Electron microscopy examination revealed that the proteinaceous material was composed of $0.7-\mu \mathrm{m}$, tightly packed myelinoid material. Further blood tests excluded known mutations in genes for surfactant proteins B and C, ABCA3 (adenosine triphosphate-binding cassette subfamily A member 3), SLAM (signalling lymphocyte-activation molecule)-associated protein and granulocyte-macrophage colony-stimulating factor (GM$\mathrm{CSF}$ ) receptor. T-cell clonality was normal and serology to human herpesvirus 6 and adenovirus were negative. Serum Ig levels, vaccine responses and lymphocyte subsets were normal, as was urine lysine.

EBV DNA was demonstrated in the lung tissue by PCR $\left(20,000\right.$ copies $\left.\cdot \mathrm{mL}^{-1}\right)$, and was also found in blood samples from day 3, 8 and 11 of the illness $\left(118,291\right.$ and 511 copies $\cdot \mathrm{mL}^{-1}$,

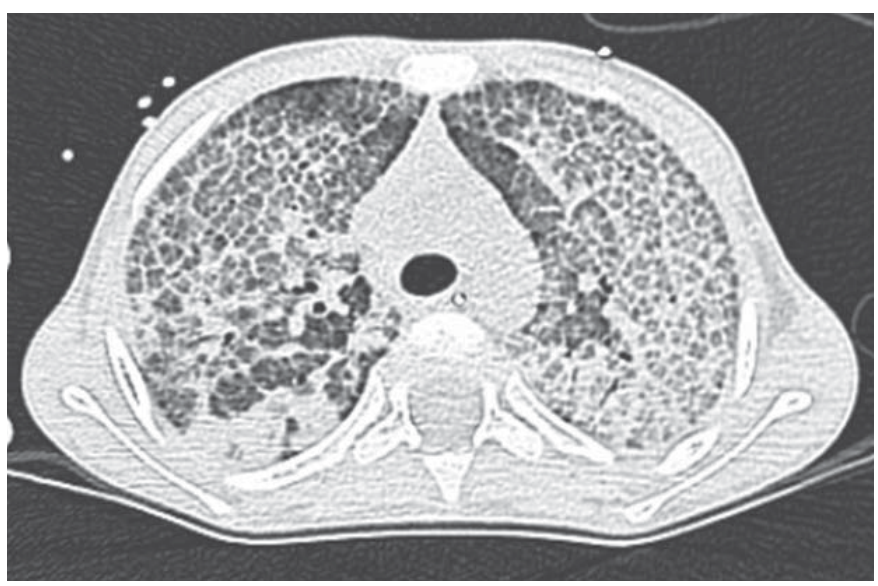

FIGURE 1. High-resolution computed tomography scan of chest, showing widespread interstitial opacities with generalised interlobular septal thickening and ground-glass opacities. 